\title{
Flow of a Hydromagnetic Fluid through Porous Media between Permeable Beds under Exponentially Decaying Pressure Gradient
}

\author{
B.G. Prasad ${ }^{1}$, Amit Kumar ${ }^{2}$ \\ ${ }^{1}$ Department of Mathematics, B N College Patna University \\ Patna-4, Bihar, India \\ e-mail:profbgprasad@gmail.com \\ ${ }^{2}$ Department of Applied Mathematics \\ Birla Institute of Technology, Deoghar Campus \\ (Mesra) Jasidih-814142, Jharkhand, India \\ e-mail:amitkumar@bitmesra.ac.in
}

(Received: 20 May 2010; revised: 31 March 2011; accepted: 1 April 2011; published online: 13 June 2011)

\begin{abstract}
An analytical solution of the flow of a hydromagnetic fluid through a porous medium between permeable beds is obtained and studied. The fluid is under an exponential decaying pressure gradient and the uniform magnetic field in a direction normal to the flow saturated porous medium is considered. Two governing equations, namely Navier-Stokes equations and Darcy's law, are employed for the flow between and through the permeable beds, respectively. Injection and suction of the fluid through lower and upper permeable beds, respectively, with same velocity are allowed in the presence of porous medium and the uniform magnetic field. The velocity field and the volume flux are calculated analytically and presented graphically for different choices of the parameters exhibiting their phenomenal nature. Additionally if we replace the exponentially decaying pressure gradient by the pulsatile one and porousity of medium tends to zero, the results match excellently with those of Malathy and Srinivas [T. Malathy and S. Srinivas, Pulsatile flow of a hydromagnetic fluid between permeable beds, Int. Comm. In Heat and Mass Transfer 35, 681-688 (2008)].

Key words: permeable beds, hydromagnetic fluid, porous medium, Darcy's law, Hartman number, exponentially decaying pressure gradient, injection and suction.
\end{abstract}

\section{INTRODUCTION}

One of the oldest chapters of the engineering sciences, the hydromagnetic fluid flow in porous media due to its rapid expanse and widespread industrial and environmental applications, is still a subject of current research interest. Special attention has been paid to the internal flows of a hydromagnetic fluid in ducts and channels filled with porous media. It was observed that generally natural channels have permeable beds such as gravel-bed rivers and generally all researchers have usually considered a permeable bed as an impermeable bed and flow resistance coefficients and velocity distributions were derived irrespective of bed porosity. Less study has been undertaken on the hydromagnetic fluid through porous media in a channel having permeable beds. The effects of interaction between the two flow regions are a non-zero velocity at the permeable boundary due to the existence of turbulent exchange of mass and momentum. An early study in this direction was presented by Wang [1] and became a fulcrum of new research in fluid dynamics using porous media. He investigated a pulsatile flow through porous medium bounded by rigid walls. Here we present a different discussion than that of Wang [1], which is actually an extension of Malathy and Srinivas [2] approach to porous media with changed unsteady pressure gradient. Jagadeeswara Pillai et al. [3] studied the aligned magnetic effects on a steady laminar, viscous, incompressible, conducting fluid under varying bed permeability and used the Darcy's law in the porous medium. Vajravelu et al. [4] examined the unsteady flow of two immiscible conducting fluids between permeable beds of different permeabilities. Thereafter Vajravelu et al. [5] discussed pulsatile flow between and through two permeable beds and showed the resulting velocity field pictorially 
for different values of the parameters. Later, Malathy and Srinivas, [2] extended it for a hydromagnetic fluid with uniform suction and injection through the permeable beds and illustrated graphically for different choices of $M$, the Hartman number. They applied the velocity field with a supposition of a uniform magnetic field in a direction normal to the flow. Chandra and Prasad [6] studied the pulsatile flow in circular tubes of varying cross section with permeable wall. Suction and injection are permissible for the fluid velocity through the wall and flow in a direction normal to the wall. Beavers and Joseph [7] investigated a slip condition at the nominal surface of the permeable bed.

Recently, MHD studies have become a useful tool for researchers due to its wide applications. Makinde [8] presented the combined effects of a transverse magnetic field and heat transfer on unsteady flow of a conducting fluid through a channel filled with saturated porous medium. Raptis et al. [9] have analyzed hydromagnetic free convection flow through a porous medium between two parallel plates. Hemida et al. [10] studied the heat transfer in laminar pulsating flow in which the pulsation amplitude does not allow flow reversed. Berman [11] investigated the laminar, two-dimensional flow of a viscous fluid driven by uniform injection (or suction) in a rectangular channel with porous walls. More applications can be found in [12-19]. Elshehawey et al. [20] considered the motion of blood as an incompressible Newtonian fluid saturating porous medium under the influence of body acceleration. Ogulu and Amos [21] examined the blood flow of a Newtonian fluid through a porous medium in a cardiovascular system under pulsatile pressure gradient. The behavior of wall permeability has been explained for different cases for the flow inside the permeable wall and is described by means of volume-averaged Navier-Stokes equations [22].

In the present work, we consider the hydromagnetic flow through a porous medium between two permeable beds. An exponential decaying pressure gradient and a uniform magnetic field in a direction normal to the flow saturated porous medium between and through permeable beds has been considered. Two governing equations NavierStokes equations and Darcy's law have been employed for the flow between and through the permeable beds, respectively. Analytical solutions to the velocity field and mass flux are obtained. These results are presented in a graphical form for different choices of $M, D a^{-1}, n, \sigma, \alpha, R$ and $t$.

\section{MATHEMATICAL ANALYSIS}

Consider the flow of a viscous incompressible fluid through and between two permeable beds apart from unit distance with different bed permeabilities $k_{1}$ and $k_{2}$. The uniform magnetic field in a direction normal to the flow through a porous medium is considered. Injection from lower permeable bed and suction through upper permeable bed of the fluid with the same velocity in the presence of porous medium and the uniform magnetic field are carried out. Two governing equations Navier-Stokes equations and Darcy's law are employed for the flow between and through the permeable beds, respectively. The flow is driven by pressure gradient which comprises a steady and an exponential decaying component in the axial direction,

$$
\frac{1}{\rho} \frac{\partial p}{\partial x}=A+B e^{-n t}
$$

where $A, B$ and $n$ are constants in which the last is positive, $\rho$ is the density, $p$ is the pressure and $t$ is the time.

The governing momentum equations and related boundary conditions for the incompressible Navier-Stokes equations can be shown to reduce to the following form for the MHD flow regime:

$$
\begin{gathered}
\frac{\partial u}{\partial x}+\frac{\partial v}{\partial y}=0 \\
\frac{\partial u}{\partial t}+V \frac{\partial u}{\partial y}=-\frac{1}{\rho} \frac{\partial p}{\partial x}+v \frac{\partial^{2} u}{\partial y^{2}}-\frac{\sigma^{*} B_{0}^{2} u}{\rho}-\frac{v}{k} u \\
0=\frac{\partial p}{\partial y}, \\
\frac{d u}{d y}=\frac{\alpha}{\sqrt{k_{1}}}\left(u_{B 1}-Q_{1}\right) \text { at } y=0, \\
\frac{u=u_{B 2} \text { at } y=h,}{d y}=-\frac{\alpha}{\sqrt{k_{2}}}\left(u_{B 2}-Q_{2}\right) \text { at } y=h,
\end{gathered}
$$

where $u$ is the velocity component in $x$-direction, $V$ the suction/injection velocity, $v$ is the kinematic viscosity, $\sigma^{*}$ is the electrical conductivity, $B_{0}$ is the magnetic field, $k$ the porosity parameter, $k_{1}$ and $k_{2}$ are the permeabilities of the lower and upper beds respectively, $\sigma_{1}=h / \sqrt{k_{1}}$ and $\sigma_{1}=h / \sqrt{k_{2}}$ are dimensionless parameters, $u_{B 1}$ and $u_{B 2}$ are the slip velocities at the lower/upper beds, respectively, $\alpha$ is the slip parameter, $h$ is the width of the channel, $Q_{1}=-k_{1} / \mu(\partial p / \partial x)$ and $Q_{2}=-k_{2} / \mu(\partial p / \partial x)$ are the Darcy's velocities, respectively.

We separate all the above equations into a steady part and an unsteady part represented by a bar $(-)$ and a tilde $(\sim)$ respectively, they become 


\section{II.1. Steady part}

$$
\begin{gathered}
\frac{\partial \bar{u}}{\partial x}=0, \\
V \frac{\partial \bar{u}}{\partial y}=-A+v \frac{\partial^{2} \bar{u}}{\partial y^{2}}-\frac{\sigma^{*} B_{0}^{2} \bar{u}}{\rho}-\frac{v}{k} \bar{u}, \\
\bar{u}=\bar{u}_{B 1} \text { at } y=0, \\
\frac{d \bar{u}}{d y}=\frac{\alpha}{\sqrt{k_{1}}}\left(\bar{u}_{B 1}-\bar{Q}_{1}\right) \text { at } y=0, \\
\frac{d \bar{u}}{d y}=-\frac{\alpha}{\sqrt{k_{2}}}\left(\bar{u}_{B 2}-\bar{Q}_{2}\right) \text { at } y=h,
\end{gathered}
$$

where

$$
\bar{Q}_{1}=-\frac{k_{1} \rho A}{\mu}, \quad \bar{Q}_{2}=-\frac{k_{2} \rho A}{\mu} .
$$

\section{II.2. Unsteady part}

$$
\begin{gathered}
\frac{\partial \tilde{u}}{\partial x}=0 \\
\frac{\partial \tilde{u}}{\partial t}+V \frac{\partial \tilde{u}}{\partial y}=-B e^{-n t}+v \frac{\partial^{2} \tilde{u}}{\partial y^{2}}-\frac{\sigma^{*} B_{0}^{2} \tilde{u}}{\rho}-\frac{v}{k} \tilde{u} \\
\tilde{u}=\tilde{u}_{B 1} \text { at } y=0 \\
\frac{d \tilde{u}}{d y}=\frac{\alpha}{\sqrt{k_{1}}}\left(\tilde{u}_{B 1}-\tilde{Q}_{1}\right) \text { at } y=0 \\
\tilde{u}=\tilde{u}_{B 2} \text { at } y=h, \\
\frac{d \tilde{u}}{d y}=-\frac{\alpha}{\sqrt{k_{2}}}\left(\tilde{u}_{B 2}-\bar{Q}_{2}\right) \text { at } y=h,
\end{gathered}
$$

where

$$
\bar{Q}_{1}=-\frac{k_{1} \rho B}{\mu} e^{-n t}, \quad \tilde{Q}_{2}=-\frac{k_{2} \rho B}{\mu} e^{-n t} .
$$

\section{DIMENSIONLESS FORM}

\section{III.1. Steady part ( )*}

$$
\begin{aligned}
& \bar{u}^{*}=\frac{\bar{u}}{\left(A_{1} h / V\right)}, \quad \bar{u}_{B 1}^{*}=\frac{\bar{u}_{B 1}}{\left(A_{1} h / V\right)}, \\
& \bar{u}_{B 2}^{*}=\frac{\bar{u}_{B 2}}{\left(A_{1} h / V\right)}, \quad \bar{Q}_{1}^{*}=\frac{\bar{Q}_{1}}{\left(A_{1} h / V\right)},
\end{aligned}
$$

$$
\begin{gathered}
\bar{Q}_{2}^{*}=\frac{V_{2}}{\left(A_{1} h / V\right)}, \quad D a=\frac{k}{h^{2}}, \\
M^{2}=\frac{\sigma^{*} B_{0}^{2} h}{\rho V}, x^{*}=\frac{x}{h}, \quad y^{*}=\frac{y}{h},
\end{gathered}
$$

where $A_{1}=-A$.

\section{III.2. Unsteady part ( )*}

$$
\begin{gathered}
\tilde{u}^{*}=\frac{u}{\left(h^{2} B_{1} / v\right)}, \quad \tilde{u}_{B 1}^{*}=\frac{\tilde{u}_{B 1}}{\left(h^{2} B_{1} / v\right)}, \\
\tilde{u}_{B 2}^{*}=\frac{u_{B 2}}{\left(h^{2} B_{1} / v\right)}, t^{*}=\frac{t}{\left(h^{2} / v\right)}, D a=\frac{k}{h^{2}},
\end{gathered}
$$

$$
M^{2}=\frac{\sigma^{*} B_{0}^{2} h}{\rho V}, \tilde{Q}_{1}^{*}=\frac{\tilde{Q}_{1}}{\left(h^{2} B_{1} / v\right)}, \tilde{Q}_{2}^{*}=\frac{\tilde{Q}_{2}}{\left(h^{2} B_{1} / v\right)},
$$

$y^{*}=\frac{y}{h}$, where $B_{1}=-B$.

Using the above dimensionless quantities, equations (2.9)-(2.20) yield: Ignoring the asterisks (*) for clarity, we obtain the dimensionless governing equation.

\section{III.3. Steady part}

$$
\begin{gathered}
\frac{\partial \bar{u}}{\partial x}=0, \\
\frac{d^{2} \bar{u}}{d y^{2}}-R \frac{d \bar{u}}{d y}-\left(M^{2} R+\frac{1}{D a}\right) \bar{u}=-R, \\
\bar{u}=\bar{u}_{B 1} \text { at } y=0, \\
\frac{d \bar{u}}{d y}=\alpha \sigma_{1}\left(\bar{u}_{B 1}-\frac{R}{\sigma_{1}^{2}}\right) \text { at } y=0, \\
\bar{u}=\bar{u}_{B 2} \text { at } y=1, \\
\frac{d \bar{u}}{d y}=-\alpha \sigma_{2}\left(\bar{u}_{B 2}-\frac{R}{\sigma_{2}^{2}}\right) \text { at } y=1,
\end{gathered}
$$

where

$$
R=\frac{V h}{v}, \quad \sigma_{1}=\frac{h}{\sqrt{k_{1}}}, \text { and } \sigma_{2}=\frac{h}{\sqrt{k_{2}}} .
$$

\section{III.4. Unsteady part}

$$
\frac{\partial \tilde{u}}{\partial x}=0
$$




$$
\frac{\partial^{2} \tilde{u}}{\partial y^{2}}-R \frac{\partial \tilde{u}}{\partial y}-\frac{\partial \tilde{u}}{\partial t}-\left(M^{2} R+\frac{1}{D a}\right) \tilde{u}=-e^{-n t} .
$$

Assuming $\tilde{u}=\tilde{f}(y) e^{-n t}$, the above equation yields

$$
\begin{gathered}
\tilde{f}^{\prime \prime}(y)-R \tilde{f}^{\prime}(y)+n \tilde{f}(y)-\left(M^{2} R+\frac{1}{D a}\right) \tilde{f}(y)=-1, \\
\Rightarrow \tilde{f}^{\prime \prime}(y)-R \tilde{f}^{\prime}(y)-\left(M^{2} R+\frac{1}{D a}-n\right) \tilde{f}=-1,
\end{gathered}
$$

and letting

$$
\tilde{u}_{B 1}=\tilde{f}_{1}(y) e^{-n t}, \quad \tilde{u}_{B 2}=\tilde{f}_{2}(y) e^{-n t} .
$$

The boundary conditions reduce to

$$
\begin{gathered}
\tilde{f}=\tilde{f}_{1} \text { at } y=0, \\
\frac{d \tilde{f}}{d y}=\alpha \sigma_{1}\left(\tilde{f}_{1}-\frac{1}{\sigma_{1}^{2}}\right) \text { at } y=0, \\
\tilde{f}=\tilde{f}_{2} \text { at } y=1, \\
\frac{d \tilde{f}}{d y}=-\alpha \sigma_{2}\left(\tilde{f}_{2}-\frac{1}{\sigma_{2}^{2}}\right) \text { at } y=1 .
\end{gathered}
$$

\section{ANALYTIC SOLUTION}

\section{IV.1. Steady part}

Using boundary conditions (3.3)-(3.6), we get the velocity field by solving (3.2) as

$$
\tilde{u}=C_{1} e^{\alpha_{1} y}+C_{2} e^{\alpha_{2} y}+\frac{R}{\left(M^{2} R+\frac{1}{D a}\right)},
$$

where

$$
\begin{gathered}
\alpha_{1,2}=\frac{1}{2}\left[R \pm \sqrt{R^{2}+4\left(M^{2} R+\frac{1}{D a}\right)}\right], \\
C_{1}=\frac{1}{e^{\alpha_{2}}-e^{\alpha_{1}}}\left[-\bar{u}_{B 2}+e^{\alpha_{2}} \bar{u}_{B 1}-\frac{R}{\left(M^{2} R+\frac{1}{D a}\right)}\left(e^{\alpha_{2}}-1\right)\right], \\
C_{2}=\frac{1}{e^{\alpha_{2}}-e^{\alpha_{1}}}\left[\bar{u}_{B 2}-e^{\alpha_{1}} \bar{u}_{B 1}-\frac{R}{\left(M^{2} R+\frac{1}{D a}\right)}\left(1-e^{\alpha_{1}}\right)\right]
\end{gathered}
$$

and the slip velocities are given by

$$
\begin{aligned}
& \bar{u}_{B 1}=\frac{D_{2} E_{2}-D_{4} E_{1}}{D_{1} D_{4}-D_{3} D_{2}}, \\
& \bar{u}_{B 2}=\frac{D_{3} E_{1}-D_{1} E_{2}}{D_{1} D_{4}-D_{3} D_{2}},
\end{aligned}
$$

where

$$
D_{1}=\frac{\alpha_{1} e^{\alpha_{2}}-\alpha_{2} e^{\alpha_{1}}}{e^{\alpha_{2}}-e^{\alpha_{1}}}-\alpha \sigma_{1}, D_{2}=\frac{\alpha_{2}-\alpha_{1}}{e^{\alpha_{2}}-e^{\alpha_{1}}},
$$

$$
D_{3}=\frac{\left(\alpha_{1}-\alpha_{2}\right) e^{\alpha_{1}+\alpha_{2}}}{e^{\alpha_{2}}-e^{\alpha_{1}}}, \quad D_{4}=\frac{\alpha_{2} e^{\alpha_{2}}-\alpha_{1} e^{\alpha_{1}}}{e^{\alpha_{2}}-e^{\alpha_{1}}}+\alpha \sigma_{2},
$$

$$
\begin{aligned}
& E_{1}=R\left[\frac{1}{\left(M^{2} R+\frac{1}{D a}\right)}\left\{\frac{\alpha_{2}\left(e^{\alpha_{1}}-1\right)}{e^{\alpha_{2}}-e^{\alpha_{1}}}-\frac{\alpha_{1}\left(e^{\alpha_{2}}-1\right)}{e^{\alpha_{2}}-e^{\alpha_{1}}}\right\}+\frac{\alpha}{\sigma_{1}}\right], \\
& E_{2}=R\left[\frac{1}{\left(M^{2} R+\frac{1}{D a}\right)}\left\{\frac{\alpha_{2} e^{\alpha_{2}}\left(e^{\alpha_{1}}-1\right)}{e^{\alpha_{2}}-e^{\alpha_{1}}}-\frac{\alpha_{1} e^{\alpha_{1}}\left(e^{\alpha_{2}}-1\right)}{e^{\alpha_{2}}-e^{\alpha_{1}}}\right\}-\frac{\alpha}{\sigma_{2}}\right] .
\end{aligned}
$$

\section{IV.2. Unsteady part}

Using boundary conditions (3.10)-(3.13), we get the velocity field by solving (3.9) as

$$
\tilde{f}(y)=A_{1} e^{\beta_{1} y}+A_{2} e^{\beta_{2} y}+\frac{1}{M^{2} R+\frac{1}{D a}-n},
$$

where

$$
\beta_{1,2}=\frac{1}{2}\left[R \pm \sqrt{R^{2}+4\left(M^{2} R+\frac{1}{D a}-n\right)}\right],
$$

$$
\begin{gathered}
A_{1}=\frac{1}{e^{\beta_{2}}-e^{\beta_{1}}}\left[-\frac{e^{\beta_{2}}-1}{M^{2} R+\frac{1}{D a}-n}-\tilde{f}_{2}+\tilde{f}_{1} e^{\beta_{2}}\right], \\
A_{2}=\frac{1}{e^{\beta_{2}}-e^{\beta_{1}}}\left[\frac{e^{\beta_{1}}-1}{M^{2} R+\frac{1}{D a}-n}+\tilde{f}_{2}-\tilde{f}_{1} e^{\beta_{1}}\right] .
\end{gathered}
$$

The unsteady part of the velocity is expressed as

$$
\tilde{u}=\tilde{f}(y) e^{-n t}
$$

and the slip velocities are given by 


$$
\begin{gathered}
\tilde{u}_{B 1}=\tilde{f}_{1} e^{-n t}=\frac{P_{2} Z_{2}-Z_{1}\left(P_{4}+Q_{2}\right)}{\left(P_{1}+Q_{1}\right)\left(P_{4}+Q_{2}\right)-P_{3} P_{2}} e^{-n t}, \\
\tilde{u}_{B 2}=\tilde{f}_{2} e^{-n t}=\frac{P_{3} Z_{1}-Z_{2}\left(P_{1}+Q_{1}\right)}{\left(P_{1}+Q_{1}\right)\left(P_{4}+Q_{2}\right)-P_{3} P_{2}} e^{-n t},
\end{gathered}
$$

where

$$
\begin{gathered}
P_{1}=\left(\beta_{1} e^{\beta_{2}}-\beta_{2} e^{\beta_{1}}\right), P_{2}=\beta_{2}-\beta_{1}, P_{3}=e^{\beta_{1}+\beta_{2}}\left(\beta_{1}-\beta_{2}\right), \\
P_{4}=\left(\beta_{2} e^{\beta_{2}}-\beta_{1} e^{\beta_{1}}\right), \\
R_{1}=\left(e^{\beta_{2}}-e^{\beta_{1}}\right), \\
Q_{1}=\alpha \sigma_{1}\left(e^{\beta_{1}}-e^{\beta_{2}}\right), Q_{2}=\alpha \sigma_{2}\left(e^{\beta_{2}}-e^{\beta_{1}}\right), \\
Z_{1}=-\frac{P_{1}+P_{2}}{M^{2} R+\frac{1}{D a}-n}+\frac{\alpha}{\sigma_{1}} R_{1}, \\
Z_{2}=-\frac{P_{3}+P_{4}}{M^{2} R+\frac{1}{D a}-n}-\frac{\alpha}{\sigma_{2}} R_{1} .
\end{gathered}
$$

\section{IV.3. Velocity of the fluid}

The fluid velocity is given by the sum of steady and unsteady part of the velocity profile

$$
\begin{aligned}
q=\bar{u}+\tilde{u} & =C_{1} e^{\alpha_{1} y}+C_{2} e^{\alpha_{2} y}+\frac{R}{\left(M^{2} R+\frac{1}{D a}\right)}+\tilde{f}(y) e^{-n t} \\
& =C_{1} e^{\alpha_{1} y}+C_{2} e^{\alpha_{2} y}+\frac{R}{\left(M^{2} R+\frac{1}{D a}\right)}+ \\
& +\left(A_{1} e^{\beta_{1} y}+A_{2} e^{\beta_{2} y}+\frac{1}{M^{2} R+\frac{1}{D a}-n}\right) e^{-n t}
\end{aligned}
$$

where unknowns are stated above.

\section{DEDUCTIONS}

V.1. The velocity field for $k_{1}=k_{2}=k\left(i . e . \sigma_{1}=\sigma_{2}=\sigma\right)$.

\section{$\underline{\text { V.1.1. Steady part }}$}

$$
\bar{u}=C_{1} e^{\alpha_{1} y}+C_{2} e^{\alpha_{2} y}+\frac{R}{\left(M^{2} R+\frac{1}{D a}\right)},
$$

where

$$
\begin{gathered}
\alpha_{1,2}=\frac{1}{2}\left[R \pm \sqrt{R^{2}+4\left(M^{2} R+\frac{1}{D a}\right)}\right] \\
C_{1}=\frac{1}{e^{\alpha_{2}}-e^{\alpha_{1}}}\left[-\bar{u}_{B 2}+e^{\alpha_{2}} \bar{u}_{B 1}-\frac{R}{\left(M^{2} R+\frac{1}{D a}\right)}\left(e^{\alpha_{2}}-1\right)\right] \\
C_{2}=\frac{1}{e^{\alpha_{2}}-e^{\alpha_{1}}}\left[\bar{u}_{B 2}-e^{\alpha_{1}} \bar{u}_{B 1}-\frac{R}{\left(M^{2} R+\frac{1}{D a}\right)}\left(1-e^{\alpha_{1}}\right)\right]
\end{gathered}
$$

and the slip velocities are given by

$$
\begin{aligned}
& \bar{u}_{B 1}=\frac{D_{2} E_{2}-D_{4} E_{1}}{D_{1} D_{4}-D_{3} D_{2}}, \\
& \bar{u}_{B 2}=\frac{D_{3} E_{1}-D_{1} E_{2}}{D_{1} D_{4}-D_{3} D_{2}},
\end{aligned}
$$

where

$$
\begin{gathered}
D_{1}=\frac{\alpha_{1} e^{\alpha_{2}}-\alpha_{2} e^{\alpha_{1}}}{e^{\alpha_{2}}-e^{\alpha_{1}}}-\alpha \sigma, \\
D_{2}=\frac{\alpha_{2}-\alpha_{1}}{e^{\alpha_{2}}-e^{\alpha_{1}}}, \\
D_{3}=\frac{\left(\alpha_{1}-\alpha_{2}\right) e^{\alpha_{1}+\alpha_{2}}}{e^{\alpha_{2}}-e^{\alpha_{1}}}, \quad D_{4}=\frac{\alpha_{2} e^{\alpha_{2}}-\alpha_{1} e^{\alpha_{1}}}{e^{\alpha_{2}}-e^{\alpha_{1}}}+\alpha \sigma, \\
E_{1}=R\left[\frac{1}{\left(M^{2} R+\frac{1}{D a}\right)}\left\{\frac{\alpha_{2}\left(e^{\alpha_{1}}-1\right)}{e^{\alpha_{2}}-e^{\alpha_{1}}}-\frac{\alpha_{1}\left(e^{\alpha_{2}}-1\right)}{e^{\alpha_{2}}-e^{\alpha_{1}}}\right\}+\frac{\alpha}{\sigma}\right], \\
E_{2}=R\left[\frac{1}{\left(M^{2} R+\frac{1}{D a}\right)}\left\{\frac{\alpha_{2} e^{\alpha_{2}}\left(e^{\alpha_{1}}-1\right)}{e^{\alpha_{2}}-e^{\alpha_{1}}}-\frac{\alpha_{1} e^{\alpha_{1}}\left(e^{\alpha_{2}}-1\right)}{e^{\alpha_{2}}-e^{\alpha_{1}}}\right\}-\frac{\alpha}{\sigma}\right] .
\end{gathered}
$$

\section{V.1.2. Unsteady part}

$$
\tilde{f}(y)=A_{1} e^{\beta_{1} y}+A_{2} e^{\beta_{2} y}+\frac{1}{M^{2} R+\frac{1}{D a}-n},
$$

where

$$
\beta_{1,2}=\frac{1}{2}\left[R \pm \sqrt{R^{2}+4\left(M^{2} R+\frac{1}{D a}-n\right)}\right]
$$




$$
\begin{gathered}
A_{1}=\frac{1}{e^{\beta_{2}}-e^{\beta_{1}}}\left[-\frac{e^{\beta_{2}}-1}{M^{2} R+\frac{1}{D a}-n}-\tilde{f}_{2}+\tilde{f}_{1} e^{\beta_{2}}\right], \\
A_{2}=\frac{1}{e^{\beta_{2}}-e^{\beta_{1}}}\left[\frac{e^{\beta_{1}}-1}{M^{2} R+\frac{1}{D a}-n}+\tilde{f}_{2}-\tilde{f}_{1} e^{\beta_{1}}\right]
\end{gathered}
$$

The slip velocities are given by

$$
\begin{gathered}
\tilde{u}_{B 1}=\tilde{f}_{1} e^{-n t}=\frac{P_{2} Z_{2}-Z_{1}\left(P_{4}+Q_{2}\right)}{\left(P_{1}+Q_{1}\right)\left(P_{4}+Q_{2}\right)-P_{3} P_{2}} e^{-n t}, \\
\tilde{u}_{B 2}=\tilde{f}_{2} e^{-n t}=\frac{P_{3} Z_{1}-Z_{2}\left(P_{1}+Q_{1}\right)}{\left(P_{1}+Q_{1}\right)\left(P_{4}+Q_{2}\right)-P_{3} P_{2}} e^{-n t},
\end{gathered}
$$

where

$$
\begin{gathered}
P_{1}=\left(\beta_{1} e^{\beta_{2}}-\beta_{2} e^{\beta_{1}}\right), \quad P_{2}=\beta_{2}-\beta_{1}, \\
P_{3}=e^{\beta_{1}+\beta_{2}}\left(\beta_{1}-\beta_{2}\right), \quad P_{4}=\left(\beta_{2} e^{\beta_{2}}-\beta_{1} e^{\beta_{1}}\right),
\end{gathered}
$$

$R_{1}=\left(e^{\beta_{2}}-e^{\beta_{1}}\right), \quad Q_{1}=\alpha \sigma\left(e^{\beta_{1}}-e^{\beta_{2}}\right), \quad Q_{2}=\alpha \sigma\left(e^{\beta_{2}}-e^{\beta_{1}}\right)$

$$
\begin{aligned}
& Z_{1}=-\frac{P_{1}+P_{2}}{M^{2} R+\frac{1}{D a}-n}+\frac{\alpha}{\sigma} R_{1}, \\
& Z_{2}=-\frac{P_{3}+P_{4}}{M^{2} R+\frac{1}{D a}-n}-\frac{\alpha}{\sigma} R_{1} .
\end{aligned}
$$

\section{V.2. The velocity field for $\boldsymbol{k}_{1}, \boldsymbol{k}_{2}$ tending to zero}

\section{V.2.1. Steady part}

$$
\begin{gathered}
\bar{u}=C_{1} e^{\alpha_{1} y}+C_{2} e^{\alpha_{2} y}+\frac{R}{M^{2} R+\frac{1}{D a}}, \\
C_{1}=\frac{1}{e^{\alpha_{2}}-e^{\alpha_{1}}}\left[-\frac{R}{M^{2} R+\frac{1}{D a}}\left(e^{\alpha_{2}}-1\right)\right], \\
C_{2}=\frac{1}{e^{\alpha_{2}}-e^{\alpha_{1}}}\left[-\frac{R}{M^{2} R+\frac{1}{D a}}\left(1-e^{\alpha_{1}}\right)\right]
\end{gathered}
$$

\footnotetext{
and the slip velocities $\bar{u}_{B 1}$ and $\bar{u}_{B 2}$ are zero.
}

\section{V.2.2. Unsteady part}

$$
\tilde{u}=\left(A_{1} e^{\beta_{1} y}+A_{2} e^{\beta_{2} y}+\frac{1}{M^{2} R+\frac{1}{D a}-n}\right) e^{-n t},
$$

where

$$
\beta_{1,2}=\frac{1}{2}\left[R \pm \sqrt{R^{2}+4\left(M^{2} R+\frac{1}{D a}-n\right.}\right]
$$

$$
\begin{gathered}
A_{1}=\frac{1}{e^{\beta_{2}}-e^{\beta_{1}}}\left[-\frac{e^{\beta_{2}}-1}{M^{2} R+\frac{1}{D a}-n}\right], \\
A_{2}=\frac{1}{e^{\beta_{2}}-e^{\beta_{1}}}\left[\frac{e^{\beta_{1}}-1}{M^{2} R+\frac{1}{D a}-n}\right] .
\end{gathered}
$$

The slip velocities $\tilde{u}_{B 1}$ and $\tilde{u}_{B 2}$ are zero.

\section{V.3. The velocity field for zero injection i.e. $R=0$}

The unsteady part of the velocity $\tilde{u}$ in (4.11) reduces to

$$
\begin{aligned}
& \tilde{u}=\mathrm{e}^{-\mathrm{nt}}\left[\frac{1}{M^{2} R+\frac{1}{D a}-n}+\right. \\
& \frac{\left(\tilde{f}_{1}-\frac{1}{M^{2} R+\frac{1}{D a}-n}\right) \sinh \beta_{1}(1-\mathrm{y})}{\sinh \beta_{1}}+
\end{aligned}
$$

$$
\left.\frac{\left(\tilde{f}_{2}-\frac{1}{M^{2} R+\frac{1}{D a}-n}\right) \sinh \beta_{1} \mathrm{y}}{\sinh \beta_{1}}\right],
$$

where

$$
\begin{aligned}
& \tilde{f}_{1}=\frac{P_{2} Z_{2}-Z_{1}\left(P_{4}+Q_{2}\right)}{\left(P_{1}+Q_{1}\right)\left(P_{4}+Q_{2}\right)-P_{3} P_{2}}, \\
& \tilde{f}_{2}=\frac{P_{3} Z_{1}-Z_{2}\left(P_{1}+Q_{1}\right)}{\left(P_{1}+Q_{1}\right)\left(P_{4}+Q_{2}\right)-P_{3} P_{2}},
\end{aligned}
$$




$$
\begin{gathered}
\beta_{1}=\frac{1}{2}\left[\sqrt{\left(M^{2} R+\frac{1}{D a}-n\right)}\right], \\
\beta_{2}=-\frac{1}{2}\left[\sqrt{\left(M^{2} R+\frac{1}{D a}-n\right)}\right], \\
P_{1}=\left(\beta_{1} e^{\beta_{2}}-\beta_{2} e^{\beta_{1}}\right), P_{2}=\beta_{2}-\beta_{1}, \\
P_{3}=e^{\beta_{1}+\beta_{2}}\left(\beta_{1}-\beta_{2}\right), P_{4}=\left(\beta_{2} e^{\beta_{2}}-\beta_{1} e^{\beta_{1}}\right), \\
R_{1}=\left(e^{\beta_{2}}-e^{\beta_{1}}\right), \quad Q_{1}=\alpha \sigma\left(e^{\beta_{1}}-e^{\beta_{2}}\right), \\
Q_{2}=\alpha \sigma\left(e^{\beta_{2}}-e^{\beta_{1}}\right), \\
Z_{1}=-\frac{P_{1}+P_{2}}{M^{2} R+\frac{1}{D a}-n}+\frac{\alpha}{\sigma} R_{1}, \\
Z_{2}=-\frac{P_{3}+P_{4}}{M^{2} R+\frac{1}{D a}-n}-\frac{\alpha}{\sigma} R_{1} .
\end{gathered}
$$

$$
=\frac{e^{-n t}}{e^{\beta_{2}}-e^{\beta_{1}}}\left[\frac{\left(\beta_{1} e^{\beta_{1}}-\beta_{2} e^{\beta_{2}}\right)+\left(\beta_{2} e^{\beta_{1}}-\beta_{1} e^{\beta_{2}}\right)}{M^{2} R+\frac{1}{D a}-n}+\right.
$$$$
\left.\left(\beta_{2}-\beta_{1}\right) \tilde{f}_{2}+\left(\beta_{1} e^{\beta_{2}}-\beta_{2} e^{\beta_{1}}\right) \tilde{f}_{1}\right]
$$$$
\tilde{\tau}_{1}=\left(\frac{\tau}{\rho h B_{1}}\right)=\frac{\partial \tilde{u}}{\partial y}
$$$$
=\left[\frac{e^{-n t}}{e^{\beta_{2}}-e^{\beta_{1}}} \frac{\left(\beta_{1} e^{\beta_{1}}-\beta_{2} e^{\beta_{2}}\right)+\left(\beta_{2} e^{\beta_{1}+\beta_{2}}-\beta_{1} e^{\beta_{2}+\beta_{1}}\right)}{M^{2} R+\frac{1}{D a}-n}+\right.
$$$$
\left.\left(\beta_{2} e^{\beta_{2}}-\beta_{1} e^{\beta_{1}}\right) \tilde{f}_{2}+\left(\beta_{1} e^{\beta_{2}+\beta_{1}}-e^{\beta_{1}+\beta_{2}}\right) \tilde{f}_{1}\right]
$$

\section{DISCUSSIONS}

\section{MASS FLOW}

The instantaneous Mass Flux $Q$ of unsteady velocity field is given by

$$
\begin{gathered}
Q=\left[\int_{0}^{1} \tilde{f}(y) d y\right] e^{-n t}= \\
\frac{1}{\beta_{1} \beta_{2}\left(e^{\beta_{2}}-e^{\beta_{1}}\right)}\left[\beta _ { 2 } e ^ { - n t } \left\{\left(e^{\beta_{1}+\beta_{2}}-e^{\beta_{2}}\right) \tilde{f}_{1}+\left(1-e^{\beta_{1}}\right) \tilde{f}_{2}+\right.\right. \\
+\beta_{1} e^{-n t}\left\{\left(e^{\beta_{1}}-e^{\beta_{1}+\beta_{2}}\right) \tilde{f}_{1}+\right. \\
\left.\left.+\left(e^{\beta_{2}}-1\right) \tilde{f_{2}}+\frac{1}{M^{2} R+\frac{1}{D a}-n}\left(e^{\beta_{1}}-1\right)\left(e^{\beta_{2}}-1\right)\right\}\right]+ \\
+\frac{e^{-n t}}{M^{2} R+\frac{1}{D a}-n} .
\end{gathered}
$$

\section{SHEAR STRESS}

We obtain the shear stress $\tilde{\tau}$ at the lower as well as upper permeable beds $\tilde{\tau}_{0}$ and $\tilde{\tau}_{1}$ using
Unsteady and steady state velocity fields and volume flux of the flow of a hydromagnetic fluid through a porous medium between permeable beds are calculated analytically and presented graphically. Steady state velocity profiles (Figs. 1, 2, 3) have been considered for various $\sigma, D a^{-1}$ and $M$ for corresponding fixed $\alpha, R, D a^{-1}$ and $M ; \alpha, R, \sigma$ and $M$ and $\alpha, R, \sigma$ and $D a^{-1}$ respectively. We have found that an increasing porosity parameter $\sigma$ (Fig. 1) and decreasing inverse Darcy number $D a^{-1}$ (Fig. 2) decreases the velocity field and the velocity field attains its maximum in the middle of the bed. Figure 3 declares that an increasing $M$ decreases the velocity profile. All are parabolic in nature attaining its maximum between the beds $(0 \leq y \leq 1)$. Unsteady state velocity profile has been plotted for various parameters in Figures 4 to 10 . It can be seen there that an increasing porosity parameter $\sigma$ decreases the velocity profile (Fig. 4) for $n=0$ (that is for constant pressure gradient) and becomes the velocity profile decreases with an increament in $n$. An increase in the Hartman number $M$ results in a reduction in the velocity field (Fig. 7) and an increasing $n$ results in a decreasing velocity profile (Figs. 7 and 8). From Figs. 9 and 10 it is noticed that the velocity profile lifts up for increasing inverse Darcy number towards upper permeable closer to upper permeable bed. On comparing Figs. 5 and 6 


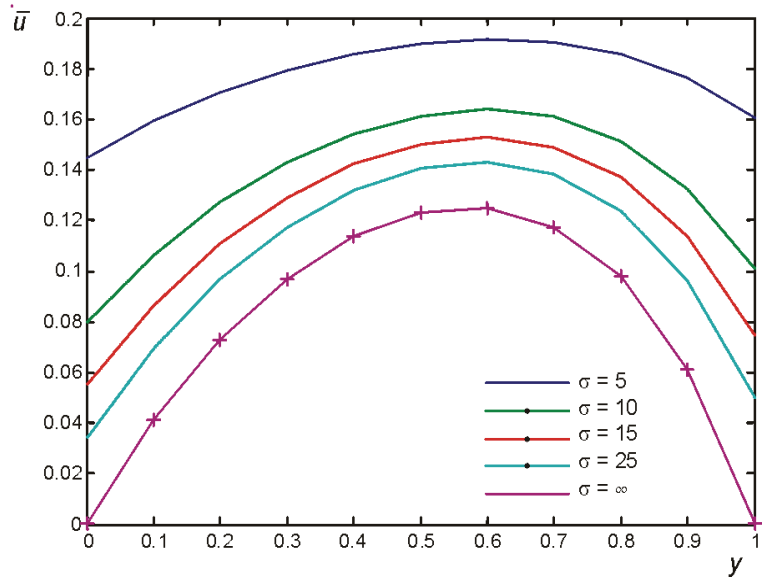

Fig. 1. Steady state velocity profile for $\alpha=0.5, R=2, \sigma=5, M=1$

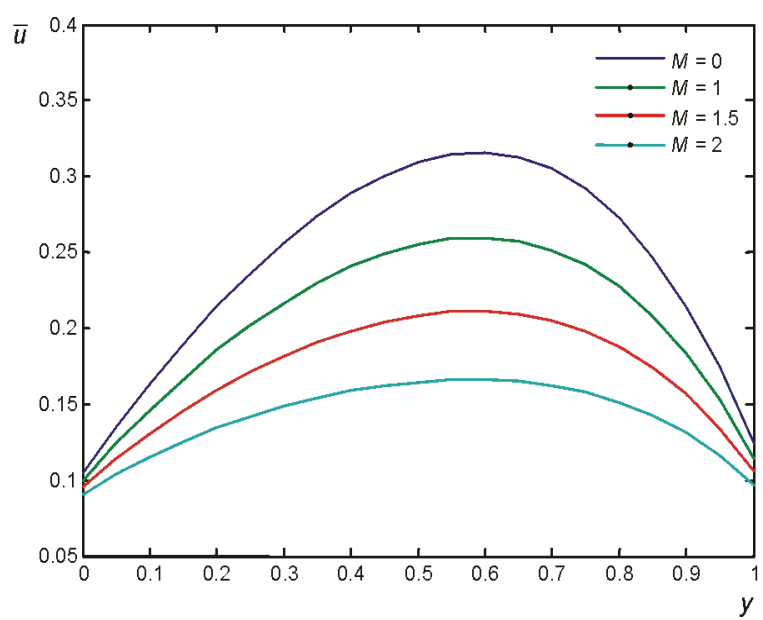

Fig. 3. Steady state velocity profile for $\alpha=0.5, R=2, \sigma=5, D a^{-1}=1$

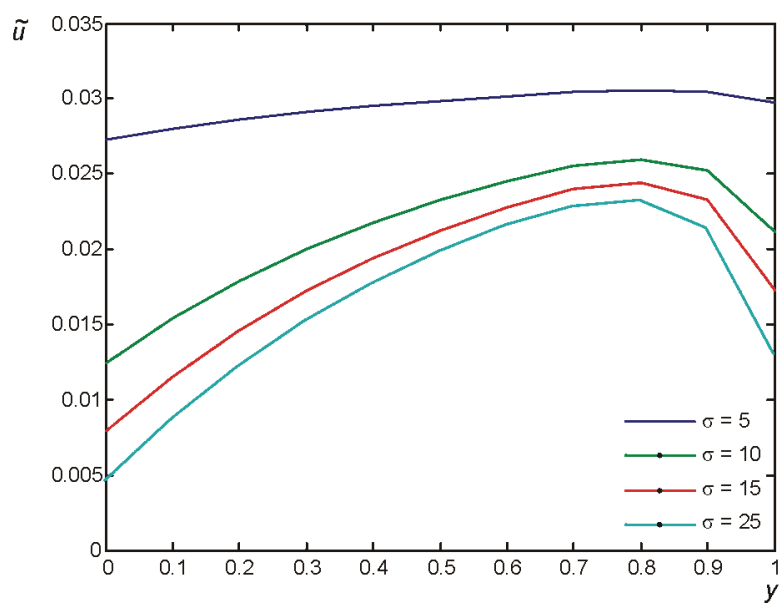

Fig. 5. Unsteady velocity profile for $M=1, D a^{-1}=0.1, R=10, n=1, t=0.5, \alpha=0.5$

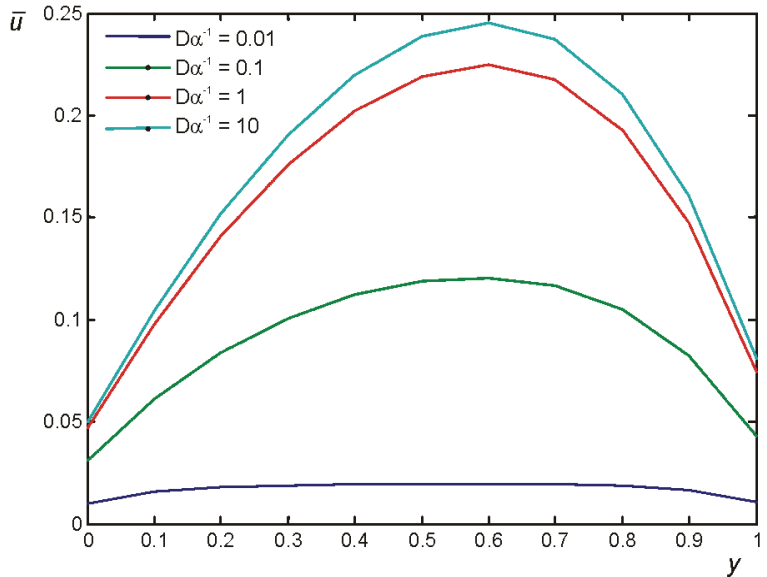

Fig. 2. Steady state velocity profile for $\alpha=0.5, R=2, \sigma=5, M=1$

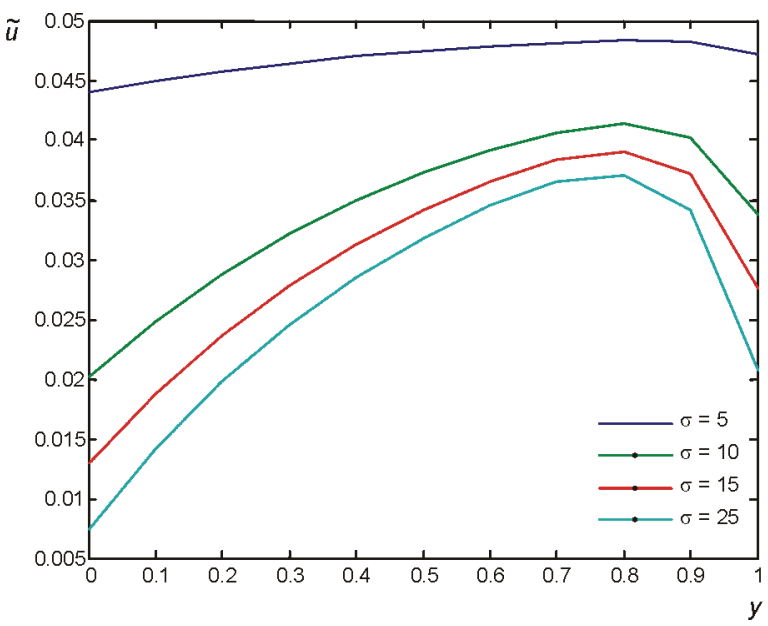

Fig. 4. Unsteady velocity profile for $M=1, D a^{-1}=0.1, R=10, n=0, \quad t=0.5, \alpha=0.5$

$\tilde{u}$

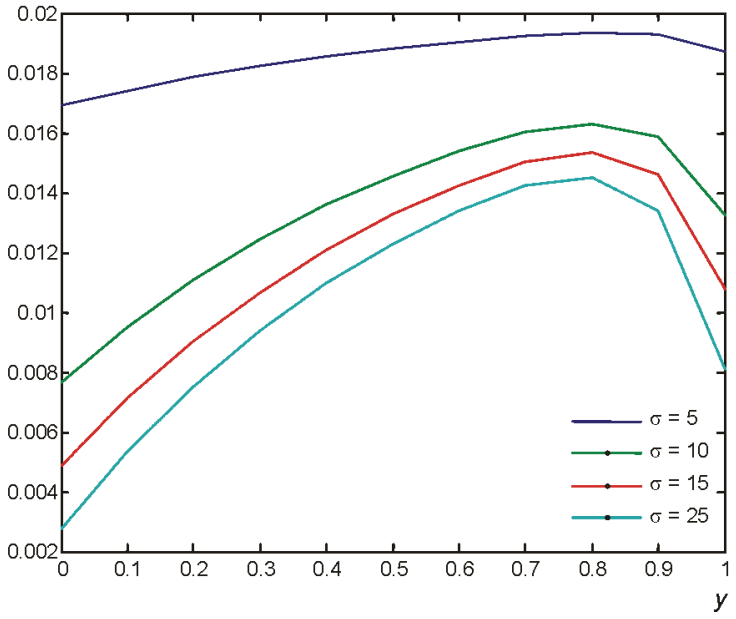

Fig. 6. Unsteady velocity profile for $M=1, D a^{-1}=0.1, R=10, n=2, t=0.5, \alpha=0.5$ 


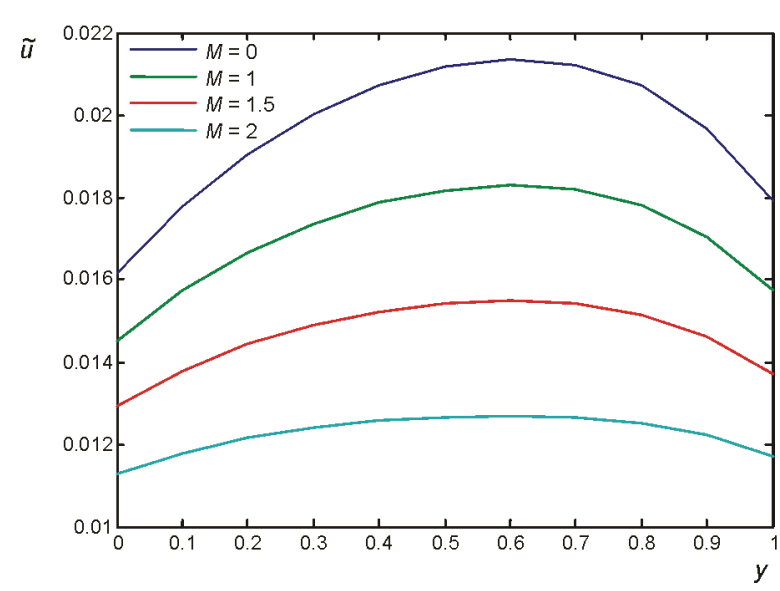

Fig. 7. Unsteady velocity profile

for $\sigma=5, D a^{-1}=0.1, R=2, n=1, t=1.5, \alpha=0.5$

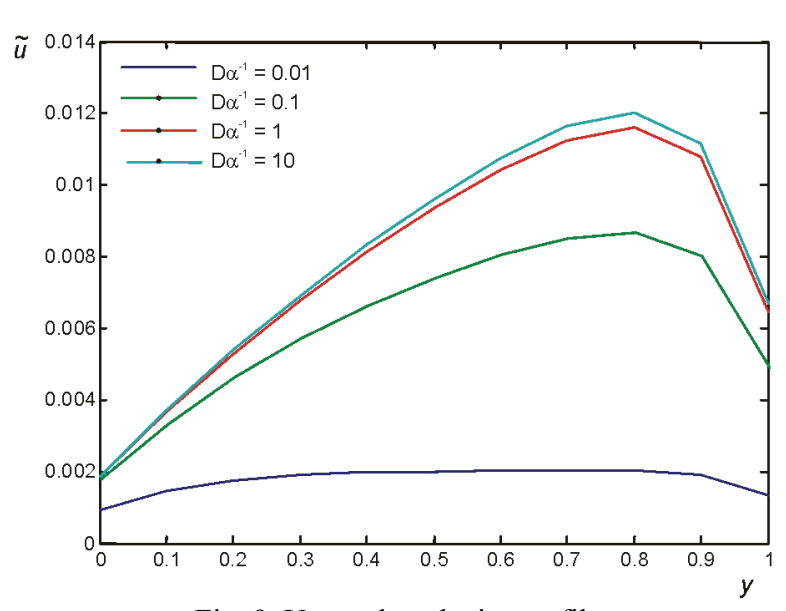

Fig. 9. Unsteady velocity profile for $\sigma=25, M=1, R=10, n=1, t=1.5, \alpha=0.5$

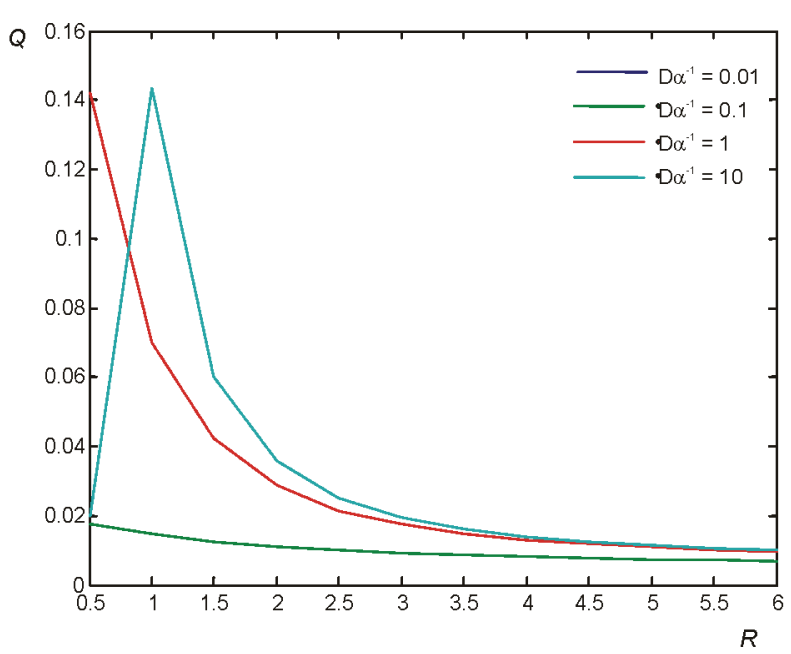

Fig. 11. Mass flux flow for $\alpha=5, n=1, t=1.5, \sigma=25$ and $M=1$

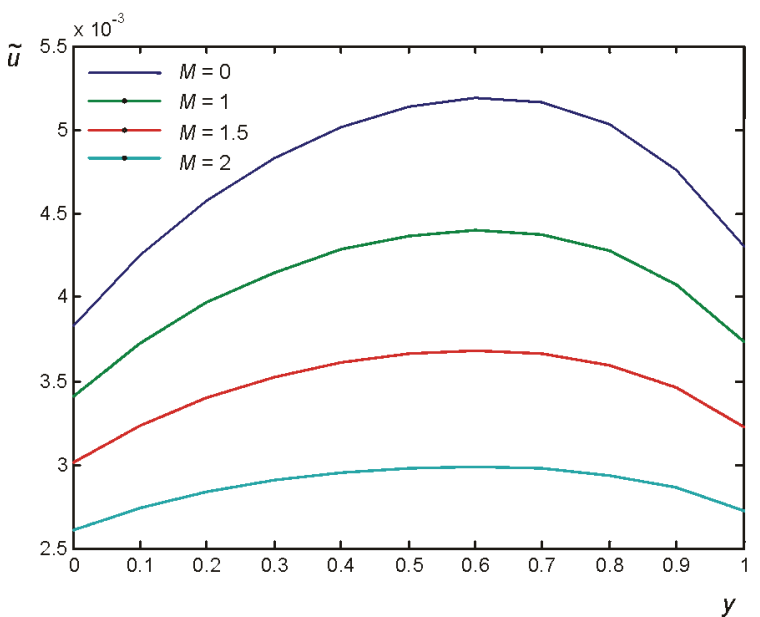

Fig. 8. Unsteady velocity profile for $\sigma=5, D a^{-1}=0.1, R=2, n=2, t=1.5, \alpha=0.5$

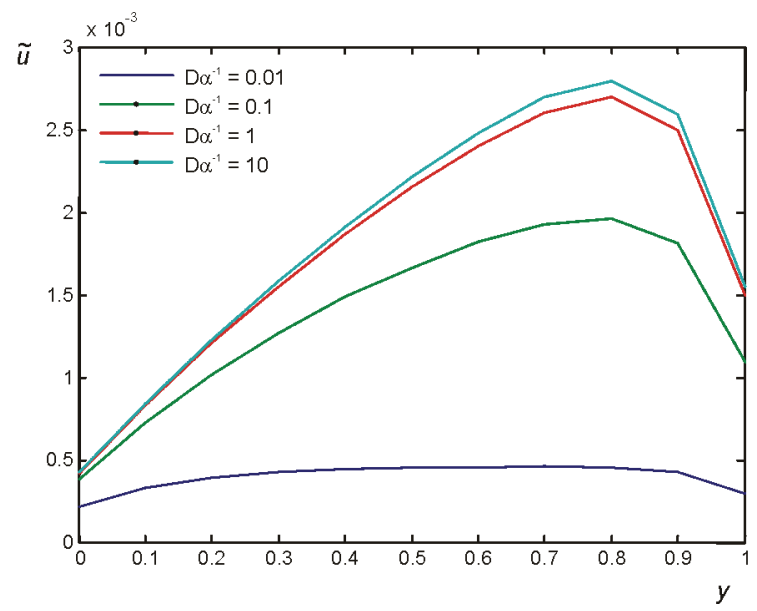

Fig. 10. Unsteady velocity profile for $\sigma=25, M=1, R=10, n=2, t=1.5, \alpha=0.5$

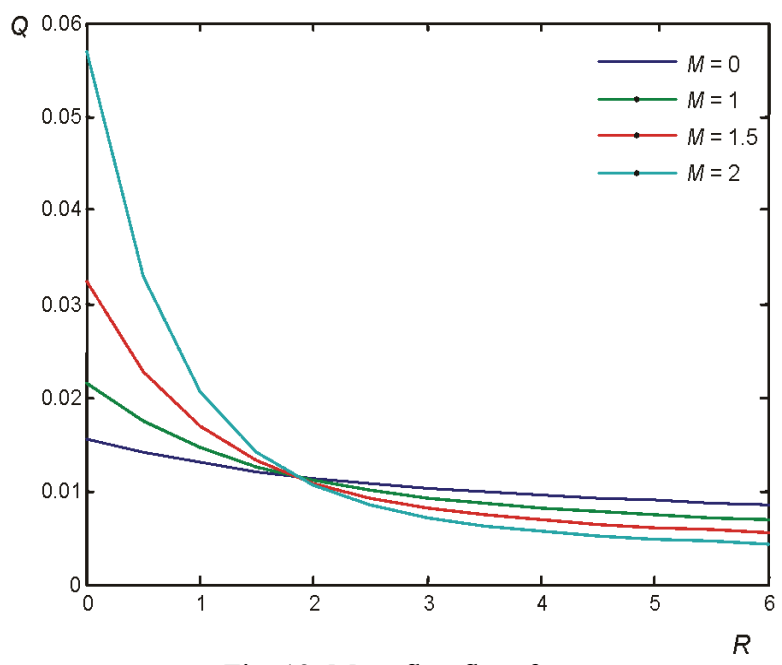

Fig. 12. Mass flux flow for $\alpha=5, D a^{-1}=0.1, n=1, t=1.5, \sigma=25$ 


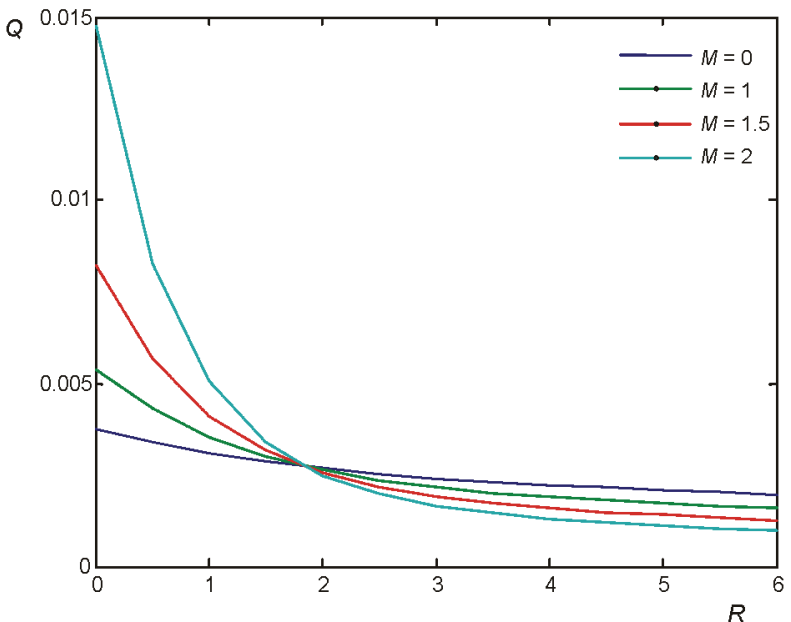

Fig. 13. Mass flux flow for $\alpha=5, D a^{-1}=0.1, n=2, t=1.5, \sigma=25$

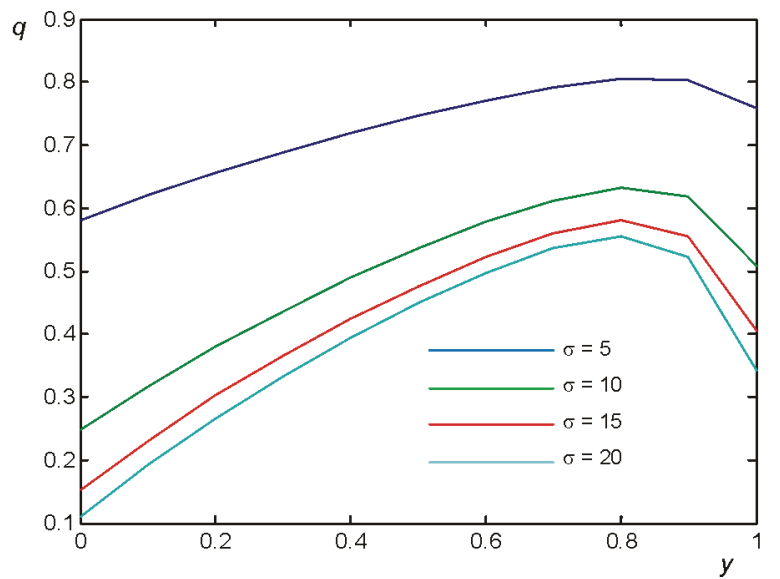

Fig. 15. Velocity profile for $D a^{-1}=0.1, M=2$, $\alpha=0.5, R=10, n=1$ and $t=1$

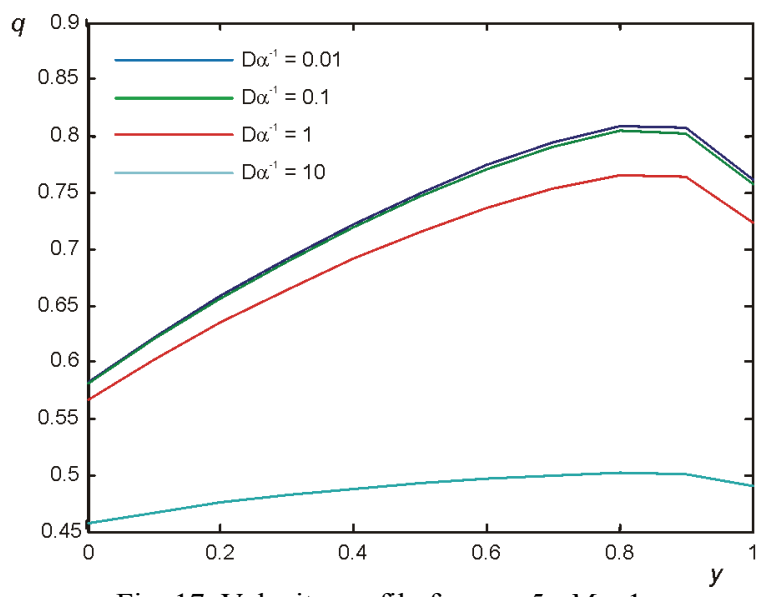

Fig. 17. Velocity profile for $\sigma=5, M=1$, $\alpha=0.5, R=10, n=1$ and $t=1$

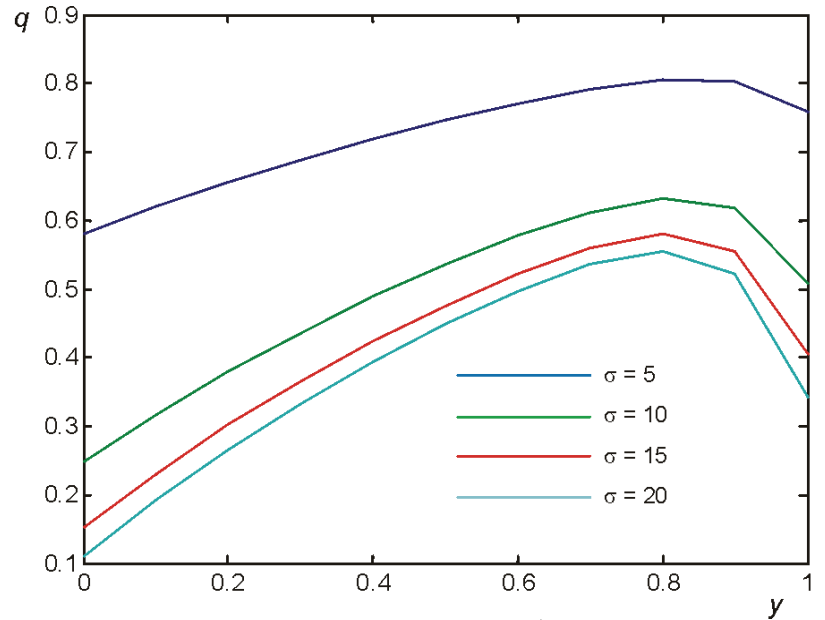

Fig. 14. Velocity profile for $D a^{-1}=0.1, M=1$, $\alpha=0.5, R=10, n=1$ and $t=1$

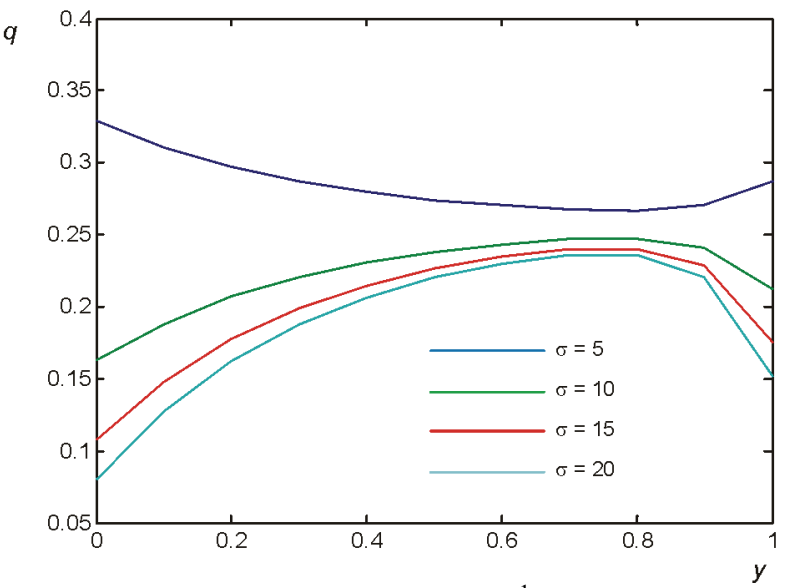

Fig. 16. Velocity profile for $D a^{-1}=0.1, M=3$, $\alpha=0.5, R=10, n=1$ and $t=1$

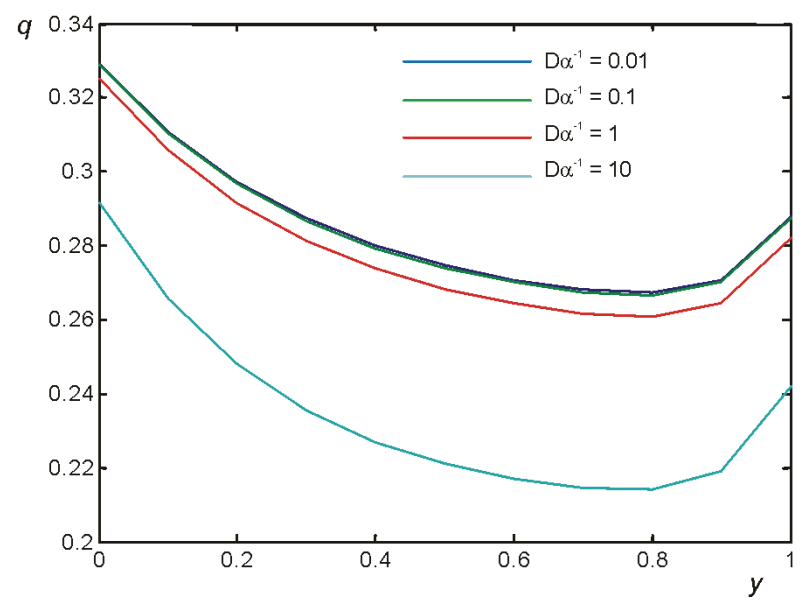

Fig. 18. Velocity profile for $\sigma=5, M=2$, $\alpha=0.5, R=10, n=1$ and $t=1$ 


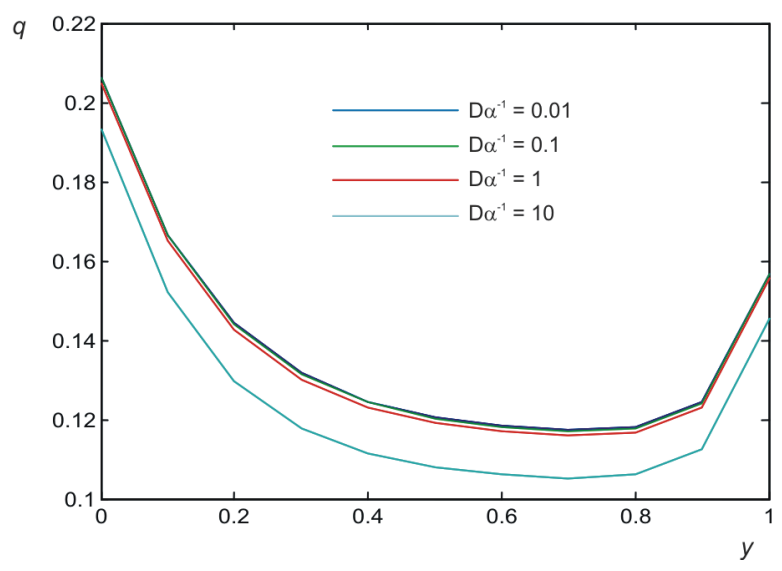

Fig. 19. Velocity profile for $\sigma=5, M=3$, $\alpha=0.5, R=10, n=1$ and $t=1$

bed. Mass flow has been presented in Figures 11, 12 and 13 for different cases. Mass flow profile (Fig. 11) illustrates that it is initially increasing and then decreasing with an increase in $R$ and the flow profile attains its maximum at $R=1$ for $D a^{-1}=10$. The smallest inverse Darcy number $\left(D a^{-1}=0.01\right)$ makes the flow field negligibly small. Figures 12 and 13 show in such a way that an increasing mass flow in the beginning decrease with an increase in $R$ for the varied Hartman number $M$ crossing the flow one another at about $(1.9,0.012)$ and $(1.9,0.003)$ respectively.

The fluid velocity profile $q$, which is the sum of steady and unsteady part of the velocity profile, has been exhibited for increasing porosity parameter $\sigma$ and the inverse Darcy number $D a^{-1}$ in the figures from 14 to 19 . The influence of the Hartman number $M$ has been displayed in the figures drawn for the velocity profile $q$. It is seen in Figures 14,15 and 16 that an increasing porosity parameter $\sigma$ decreases the fluid velocity $q$ and their nature depends upon the variation of the Hartman number $M$. For $M=1$ the velocity increases with an increase of $y$ and approaches its maximum peak at about $y=0.8$ near the upper permeable bed and then decreases but in the next figures (Figs. 15 and 16), the behavior of the fluid velocities for $\sigma=5$ changes and becomes just opposite to those for $\sigma=10,15$ and 20. These figures (14, 15 and 16) show that increasing Hartman number $M$ decreases the velocity profile $q$. The last three figures (Figs. 17, 18 and 19) have been depicted for an increasing inverse Darcy number $D a^{-1}$ against $y$. Figure 17 shows that an increasing inverse Darcy number decreases the velocity $q$. Here the velocity profile increases with increase in $y$ and reaches its maximum in the region $0.8 \leq y \leq 0.9$ close to upper bed then decreases. Hence, once again, the velocity is at its peak near the upper permeable bed. Figures 18 and 19 have been presented for $M=2$ and 3, which make the velocity curve concavity upward and are just opposite to the previous one. In this case the fluid velocity decreases first and reaches its minimum near the upper permeable bed at about $y=0.8$ then increases. It is noticed that the fluid velocity near the lower permeable bed is greater than those near the upper permeable bed. All the graphs have been drawn with the help of Matlab.

\section{References}

[1] Y.C. Wang, Pulsatile flow in a porous channel. J. Appl. Mech. 38, 553-555 (1971).

[2] T. Malathy, S. Srinivas, Pulsatile flow of a hydromagnetic fluid between permeable beds. Int. Comm. In Heat and Mass Transfer 35 681-688 (2008).

[3] K. Jagadeeswara Pillai, S. Vijaya Kumar, Varma, M. Syam $\mathrm{Babu}$, Aligned magnetic effects through varying permeable bed. Proceedings Mathematical Sciences 96, 61-69 (2008).

[4] K. Vajravelu, P.V. Arunachalam, S. Sreenadh, Unsteady flow of two immiscible conducting fluids between two permeable beds, J. Math. Anal. Appl. 196 1105-1116 (1995).

[5] K. Vajravelu, K. Ramesh, S. Sreenadh, P.V. Arunachalam, Pulsatile flow between permeable beds. Int. J. Non-Linear Mech. 38, 999-1005 (2003).

[6] P. Chandra, J.S.V.R.K. Prasad, Pulsatile flow in circular tubes of varying cross-section with suction/injection, J. Aust. Math. Soc. Ser. B 35, 366-381 (1994).

[7] G.S. Beavers, D.D. Joseph, Boundary conditions at a naturally permeable wall. J. Fluid Mech. 30, 197-206 (1967).

[8] O.D. Makinde, P.Y. Mhone, Heat transfer to MHD oscillatory flow in a channel filled with porous medium. Rom. J. Phys. 50, 931-938 (2005).

[9] A. Raptis, C. Massalas, G. Tzivanidis, Hydromagnetic free convection flow through a porous medium between two parallel plates. Phys. Lett. 90A, 288-289 (1982).

[10] H.N. Hemida, M.N. Sabry, A. Abdel-Rahim, H. Mansour, Theoretical analysis of heat transfer in laminar pulsating flow. Int. J. Heat Mass Transfer 45, 1767-1780 (2002).

[11] A.S. Berman, Laminar flow in channels with porous walls. J. Appl. Phys. 24, 1232-1235 (1953).

[12] S. Srinivas, T. Malathy, P.L. Sachdev, On pulsatile hydromagnetic flow of an Oldroyd fluid with heat transfer. Eng. Trans. 55 (1), 79-94 (2007).

[13] H.A. Attia, Unsteady hydromagnetic Couette flow of a dusty fluid with temperature dependent viscosity and thermal conductivity under exponential decaying pressure gradient. Comm. in Nonlinear Sc. And Num. Simulation 13, 10771088 (2008).

[14] A.R. Rao, K.S. Deshikachar, MHD oscillatory flow of blood through channels of variable cross section. Int. J. Eng. Sci. 24, 1615-1628 (1986).

[15] G. Radhakrishnamacharya, M.K. Maiti, Heat transfer to pulsatile flow in a porous channel. Int. J. Heat Mass Transfer 20, 171-173 (1977).

[16] K.R. Rajagopal, L. Tao, Mechanics of mixtures, Series on Advances in Mathematics for Applied Sciences. Vol. 35, World Scientiffic Publishiing Co., Inc, River Edge, NJ, 1995. 
[17] J.R. Sellars, Laminar flow in channels with porous walls at high suction Reynolds numbers. J. Appl. Phys. 26, 489-490 (1955).

[18] S.W. Yuan, Further investigation in laminar flow in channels with porous walls. J. Appl. Phys. 27, 267-269 (1956).

[19] M. Morduchow, On laminar flow through a channel or tube with injection-application of method of averages. Quart. J. Appl. Math. 14, 361-368 (1956).

[20] E.F. Elshehawey, E.M.E. Elbarbary, N.A.S. A88, M. ElShahed, Pulsatile flow of blood through a porous medium under periodic body acceleration. Int. J. Theoret. Phys. 39, 181-188 (2000).

[21] A. Ogulu, E. Amos, Modeling pulsatile blood flow within a homogeneous porous bed in the presence of a uniform magnetic field and time-dependent suction. Int. Comm. Heat Mass 34, 989-995 (2007).

[22] W.P. Breugem, B.J. Boersma, R.E. Uittenbogaard, The influence of wall permeability on turbulent channel flow Journal of Fluid Mechanics 562, 35 (2006).

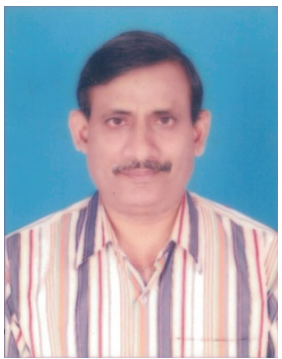

Dr. Balgangadhar Prasad, 28 September, 1954, Ph.D. in Applied Mathematics, Banaras Hindu University, India, 1980; Nationality: Indian; E-mail: profbgprasad@gmail.com; Membership: Bihar Mathematical Society; Current affiliation: Professor and Head of Department of Mathematics, BN College, Patna University, Patna, India; Professional: Fluid Dynamics, Operations Research, Functional Analysis; Publications: 3 papers in peer reviewed Journals and 4 seminar/conference presentations, 1 book (Functional Analysis); Honours: national merit scholarship for pursuing M.Sc.

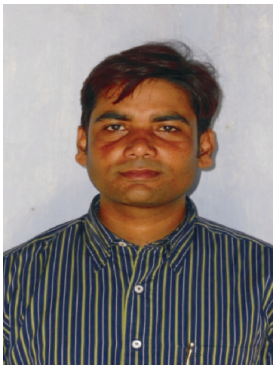

Dr. Amit Kumar, 20 June, 1980, M.Sc in Mathematics, Patna University, Bihar, India, 2005; M.Phil., Algappa University, Kadaikudi, Tamilnadu, India, 2007; Ph. D. in Fluid Dynamics on the topic "Solution of some flow problems in viscous incompressible fluids", Patna University, India, 2011; Current affiliation: Lecturer in Birla Institute of Technology (Mesra) Deoghar Campus, India, 2007 till date; Nationality: Indian; E-mail: amitkumar@bitmesra.ac.in; Professional: Fluid Dynamics, Publications: 2 papers in peer reviewed Journals and 2 seminar/conference attended. 